\title{
Disparate Connectivity for Structural and Functional Networks is Revealed When Physical Location of the Connected Nodes is Considered
}

\author{
José Ángel Pineda-Pardo • Kenia Martínez • \\ Ana Beatriz Solana $\cdot$ Juan Antonio Hernández-Tamames • \\ Roberto Colom • Francisco del Pozo
}

Received: 11 April 2014/ Accepted: 25 August 2014

(C) Springer Science+Business Media New York 2014

\begin{abstract}
Macroscopic brain networks have been widely described with the manifold of metrics available using graph theory. However, most analyses do not incorporate information about the physical position of network nodes. Here, we provide a multimodal macroscopic network characterization while considering the physical positions of nodes. To do so, we examined anatomical and functional macroscopic brain networks in a sample of twenty healthy subjects. Anatomical networks are obtained with a graph based tractography algorithm from diffusion-weighted
\end{abstract}

J. Á. Pineda-Pardo $(\bowtie) \cdot$ A. B. Solana

J. A. Hernández-Tamames · F. d. Pozo

Laboratory of Neuroimaging, Centre for Biomedical

Technology, Universidad Politécnica de Madrid, Campus de

Montegancedo, 28223, Pozuelo De Alarcón, Spain

e-mail: joseangel.pineda@ctb.upm.es

A. B. Solana

e-mail: anabea.solana@ctb.upm.es

J. A. Hernández-Tamames

e-mail: juan.tamames@urjc.es

F. d. Pozo

e-mail: francisco.delpozo@ctb.upm.es

K. Martínez $\cdot$ R. Colom

Department of Biological and Health Psychology, Universidad

Autónoma de Madrid, Ciudad Universitaria de Cantoblanco,

28049 Madrid, Spain

e-mail: kenia.martinez.r@gmail.com

R. Colom

e-mail: roberto.colom@uam.es

J. A. Hernández-Tamames

Department of Electronics Technology, Universidad Rey Juan

Carlos, Calle Tulipán S/N, 28933 Móstoles, Spain magnetic resonance images (DW-MRI). Anatomical connections identified via DW-MRI provided probabilistic constraints for determining the connectedness of 90 different brain areas. Functional networks are derived from temporal linear correlations between blood-oxygenation level-dependent signals derived from the same brain areas. Rentian Scaling analysis, a technique adapted from verylarge-scale integration circuits analyses, shows that functional networks are more random and less optimized than the anatomical networks. We also provide a new metric that allows quantifying the global connectivity arrangements for both structural and functional networks. While the functional networks show a higher contribution of inter-hemispheric connections, the anatomical networks highest connections are identified in a dorsal-ventral arrangement. These results indicate that anatomical and functional networks present different connectivity organizations that can only be identified when the physical locations of the nodes are included in the analysis.

Keywords Brain connectivity - Resting state fMRI Diffusion tensor tractography · Graph theory · Rentian scaling

$\begin{array}{ll}\text { Abbrevation } \\ \text { FC } & \text { Functional connectivity } \\ \text { AC } & \text { Anatomical connectivity } \\ \text { MRI } & \text { Magnetic resonance imaging } \\ \text { DW-MRI } & \begin{array}{l}\text { Diffusion weighted magnetic resonance } \\ \text { imaging }\end{array} \\ \text { BOLD } & \text { Blood-oxygenation level-dependent } \\ \text { fMRI } & \text { Functional magnetic resonance imaging } \\ \text { rsfMRI } & \text { Resting state functional magnetic resonance } \\ & \text { imaging } \\ \text { TR } & \text { Repetition time }\end{array}$




$\begin{array}{ll}\text { TE } & \text { Echo time } \\ \text { FoV } & \text { Field of view } \\ \text { GM } & \text { Grey matter } \\ \text { CSF } & \text { Cerebrospinal fluid } \\ \text { WM } & \text { White matter } \\ \text { ACP } & \text { Anatomical connection probability } \\ \text { tRS } & \text { Topological rentian scaling } \\ \text { pRS } & \text { Physical rentian scaling } \\ \text { mRS } & \text { Minimum rentian scaling } \\ \text { rtRE } & \text { Random topological rentian scaling } \\ \text { rpRE } & \text { Random physical rentian scaling } \\ \text { IH } & \text { Inter-hemispheric } \\ \text { AP } & \text { Anterior-posterior } \\ \text { DV } & \text { Dorsal-ventral } \\ \text { ROI } & \text { Region of interest } \\ \text { EEG } & \text { Electroencephalography } \\ \text { MEG } & \text { Magnetoencephalography }\end{array}$

\section{Introduction}

There has been recent emphasis in the neurosciences in determining how large scale brain networks process information in an integrated manner (Bullmore and Sporns 2009). Functional connectivity (FC), commonly defined as the statistical dependence between clusters of neuronal activity, is considered to be an information transfer mechanism (Singer 1993). This FC is physically instantiated via distributed anatomical connectivity (AC) which can be non-invasively quantified by means of diffusion weighted MRI (Hagmann et al. 2006). Although it is clear that $\mathrm{FC}$ relies upon $\mathrm{AC}$, the exact relation that exists between the anatomical and FC in networks is still an open question (see Damoiseaux and Greicius 2009; Guye et al. 2010; Rykhlevskaia et al. 2008 for reviews). Early research on this topic focused on relating $\mathrm{FC}$ to $\mathrm{AC}$ within few regions in the brain (Koch et al. 2002; Guye et al. 2003; Boorman et al. 2007; Cohen et al. 2008; Zhou et al. 2008; Takahashi et al. 2008). There is also strong evidence that anatomical networks support the formation of functional patterns of resting state connectivity (van den Heuvel et al. 2008; Skudlarski et al. 2008; Greicius et al. 2009; van den Heuvel et al. 2009; Teipel et al. 2010). Disruption of this FC-AC dependence has been thought to result in significant behavioural deficits related to disconnection syndromes such as schizophrenia (Skudlarski et al. 2010; Camchong et al. 2011).

Networks science provides a framework to examine FC and AC topologies for the whole brain (Rubinov and Sporns 2010). From a local network perspective it has been found that there is a "rich club" of nodes, i.e. a group of nodes that present high strength and centrality within the macroscopic brain networks (e.g., superior frontal cortex, insula). This group of nodes has been identified in both modalities (FC-AC) of networks (Hagmann et al. 2008), and is responsible for a disproportionate consumption of energetic resources (Collin et al. 2013). From a global network perspective some investigations have related a large manifold of network properties between anatomical and functional networks (Honey et al. 2007; Guye et al. 2010; Hosseini and Kesler 2013). Hosseini and Kesler demonstrated that AC networks obtained from cortical volume correlations were more small-world than the FC networks from linear correlations, and hence AC networks tended to be more random. Although these descriptions of multi-modal brain networks in terms of graph metrics are not uncommon in the literature, most studies characterize the networks topologically, without considering the embedding space for these models. Because macroscopic brain networks are composed of physically separate nodes, we think that it is necessary to consider these variables (e.g. three dimensional coordinates) in order to achieve a full network characterization. Furthermore we hypothesize that the inclusion of another dimension, i.e. the physical location of the nodes, in the characterization of brain networks, will lead to a better understanding of the organization in $\mathrm{AC}$ and $\mathrm{FC}$ networks.

Here we characterize FC-AC networks in the same set of subjects. FC was computed through Pearson linear correlations between pairs of fMRI temporal oscillations in resting state. AC was determined using a graph based tractography method (Iturria-Medina et al. 2007). We defined a set of 90 nodes using the automated anatomical labelling atlas (Tzourio-Mazoyer et al. 2002), and we used these 90 regions as nodes in our networks. These multimodal, FC-AC, networks were then characterized in a new framework, i.e. including the spatial coordinates of the network's nodes in the analyses.

We applied an adapted methodology from VLSI ("very large scale integration") circuits, i.e. Rentian Scaling (RS), to the study of the brain networks organization. RS analysis, which was first applied to the characterization of anatomical networks, (Bassett et al. 2010), allows one to quantify the degree of optimization of a network. The current study is the first to examine the RS for both FC-AC networks in the same sample of subjects.

It was a second goal to outline a procedure for quantifying the directionality of the network's links. Jbabdi and colleagues have argued that the spatial arrangements of the anatomical networks provide complementary information to the one provided by traditional graph metrics (Jbabdi et al. 2013). Although they focused on microscopic and histological arrangements, features of the macroscopic spatial arrangement have been related to brain maturation (Fair et al. 2008) and ageing (Andrews-Hanna et al. 2007). 
Based upon this literature we provide a characterization of this spatial arrangement in healthy macroscopic anatomical networks, and compare it with the rsFC arrangements and propose a metric called network directionality (ND). ND quantifies the magnitude of the network strength in the three main axes, inter-hemispheric (IH), dorsal-ventral and anterior-posterior providing a vector of three weights that represent the influence of the global connectivity on each of these main axes.

\section{Materials and Methods}

\section{Data Acquisition}

The sample comprised twenty right-handed young females (mean age: 18.9, S.D.: 2.6). They were psychology undergraduates and presented no history of psychiatric or neurological illness. Written informed consent was obtained in accordance with regulations of Fundación CIEN-Fundación Reina Sofía (Madrid). The local ethical committee approved the study.

Each participant was scanned on a 3T General Electric MR scanner (General Electric Healthcare, Farfield, CT), using a whole-body radiofrequency coil for signal excitation and quadrature 8-channel brain coil for reception. The acquisition protocol consisted of: (A) high resolution 3D T1weighted Gradient Echo-SPGR with $\mathrm{TR}=9.2 \mathrm{~ms}$, $\mathrm{TE}=4.2 \mathrm{~ms}$, flip angle $80^{\circ}$, matrix $=260 \times 260$, slice thickness $=1 \mathrm{~mm}$, preparation time $=650 \mathrm{~ms}$, obtaining a complete volume with 154 sagittal slices, (B) six minutes resting state fMRI scan with Gradient-Echo EPI sequence with $\mathrm{TR}=3,000 \mathrm{~ms}, \mathrm{TE}=28.1 \mathrm{~ms}$, flip angle $=90^{\circ}$, $\mathrm{NEX}=1, \quad$ matrix $=128 \times 128, \quad \mathrm{FoV}=24 \mathrm{~cm}$, slice thickness $=2.4 \mathrm{~mm}$, interslice gap $=0.3 \mathrm{~mm}$, yielding to 120 fMRI volumes, and (C) 26 diffusion weighted images (DWI) and two $\mathrm{b}=0 \mathrm{~s} / \mathrm{mm}^{2}\left(\mathrm{~b}_{0}\right)$ images with single-shot echo planar sequence with $\mathrm{TR}=8,200 \mathrm{~ms}, \mathrm{TE}=89.8 \mathrm{~ms}$, $\mathrm{NEX}=2, \quad$ matrix $=128 \times 128, \quad \mathrm{FoV}=24 \mathrm{~cm}$, slice thickness $=2.4 \mathrm{~mm}$, interslice gap $=0.3 \mathrm{~mm}$, in-plane resolution $=2 \mathrm{~mm}^{2}$, b value $=1,000 \mathrm{~s} / \mathrm{mm}^{2}$.

\section{Data Preprocessing}

\section{Parcellation}

The T1-weighted images were automatically segmented using the IBASPM toolbox (available at http://www.fil.ion. ucl.ac.uk/spm/ext/ \#23IBASPM). IBASPM uses the anatomically labeled template image (Tzourio-Mazoyer et al. 2002), and segments each brain hemisphere into 45 gray matter (GM) regions of interest (ROI). IBASPM provides outputs regarding the probabilistic tissue maps for GM, cerebro-spinal fluid (CSF) and white matter (WM) probabilistic maps that were obtained using the segment function from SPM8 (available at http://www.fil.ion.ucl.ac.uk/spm/ software/spm8/). The T1-weighted images were then coregistered to the $b_{0}$ and average fMRI images using a normalized mutual information method (Ashburner 2007). Thus, we obtained the affine transformations from $\mathrm{T} 1$ to DWI and from T1 to fMRI native spaces. These transformations were applied for the 90 ROIs and to the probabilistic tissue maps for both DWI and fMRI datasets.

\section{Anatomical Connectivity}

DWI 4D images were pre-processed using FMRIB's Diffusion Toolbox (FDT). Correction for Eddy currents distortion and head motion was performed with the eddycorrect function in FDT, taking as reference image the first $b_{0}$ volume. Non-brain tissue from the average $b_{0}$ image was removed using the FMRIB's Brain Extraction Toolbox, BET (Smith 2002). The brain mask was applied to the rest of the DWI images. Diffusion tensor images (DTI) were built with the dtifit function from FDT. We chose a weighted least squares regression for the tensor fitting. DTI's together with GM-WM probabilistic maps were set up as inputs to the tracking algorithm defined in (IturriaMedina et al. 2007). This algorithm characterizes AC between different brain GM structures. It consists on the following steps (see Iturria-Medina et al. (2007) for a deeper description of the method)

- The brain is defined as a non-directed weighted graph where each GM or WM voxel represents a node.

- The link weight between two adjacent nodes is determined by the integral of the diffusion tensor in the solid angle around the direction that connects the two nodes.

- The most likely paths (maximum sequence of weights) for all pair of nodes are identified using Dijkstra algorithm (Dijkstra 1959).

- The connectivity between every two nodes is finally defined as the minimum link weight that is found in the most probable path between them. In this manner connectivities are not biased by distance. As illustration, Fig. 1 shows the AC map using the left supplementary motor area as seed region.

- For each node in the boundary of an ROI we compute its connectivity with all the nodes in all the boundaries of the remaining's ROIs.

- The anatomical connection probability (ACP) (IturriaMedina et al. 2007) between two ROIs is defined as the maximum node to node connectivity between the boundaries of the ROIs. 
Fig. 1 Anatomical connectivity map with seed in the leftsupplementary-motor area. The color overlaid in the T1 image indicates the maximum probability of being connected to any of the nodes (voxels) of the seed region
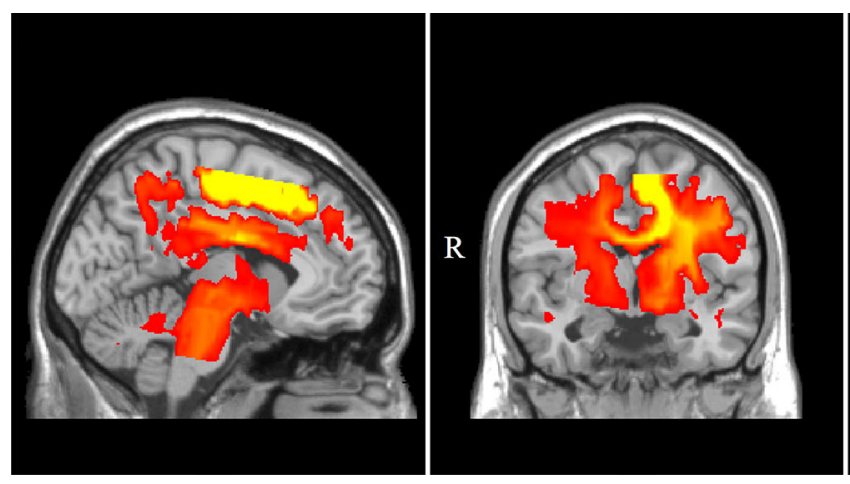

Following this pipeline and using the 90 ROIs previously defined we built one ACP matrix $(90 \times 90$ weighted network) per subject. ACP will be referred to as AC for the remainder of the paper.

\section{Resting-State Functional Connectivity}

Each fMRI data set was initially corrected for slice timing and for image displacements due to motion with SPM8 (http://www.fil.ion.ucl.ac.uk/spm/software/spm8/). The time series for the 90 regions were obtained by averaging the fMRI time series over all voxels in each ROI, thus making a smoothing step unnecessary in the pre-processing. These regional time series were band-pass filtered $(0.01-0.09 \mathrm{~Hz})$ and linearly detrended. The time series of the WM and CSF were averaged and, together with the six motion correction estimation residuals, regressed out linearly from the GM regional time series. Twenty functional networks were obtained performing Pearson linear correlations between all 90 residuals from the linear regression. We include only positive correlations with statistical significance of $p<$ $1 \mathrm{e}-03$ consistent with prior experiments (Honey et al. 2009).

\section{Data Analysis}

\section{Networks Definition}

Graph theory was used to create macroscopic brain networks. Each network constitutes a non-directed weighted graph. $\mathrm{N}$ represents the 90 brain GM ROIs. W represents the weights of the connections between the nodes in the network. rsFC networks just considered the significant positive correlations as functional links.

\section{Rentian Scaling}

The optimization of the organization of the networks has been studied by computing the topological (tRS) and physical Rentian Scaling (pRS) of the networks (Christie and Stroobandt 2000). First, the networks were thresholded, leaving just the $10 \%$ of the higher weights. This threshold was selected in agreement with Gong et al. (2009), which reported a stable density of around $\sim 11 \%$ of the possible links for DTI-tractography anatomical networks. For the sake of comparability we applied this threshold to both $\mathrm{AC}$ and rsFC networks

The $p R S$ quantification is performed in two steps (see Fig. 2a, b):

- The three-dimensional space of the brain network is covered with a random sized cube. The maximum size for this cube is the smallest size that covers the whole network, whereas the minimum size is not constrained.

- The number of links, W, that cross (only once) the walls of the cube and the number of inside nodes, $\mathrm{K}$, are counted.

This iterative process was repeated $\mathrm{L}=5000$ times. pRS exists if the function $\mathrm{W}(\mathrm{K})$ follows a power law as $W(K) \approx K^{B}$ (see Fig. 2c). In this case $B$ is called physical Rent's Exponent, pRE (Christie and Stroobandt 2000). pRE is the slope of $\mathrm{W}(\mathrm{K})$ in the $\log -\log$ scale in the linear regime using least-squares fitting.

tRS is calculated similarly to pRS. In this case the network is iteratively divided into M sub-networks, where M varies from two to a maximum number of sub-networks, which we defined to be 30 . The sub-networks are defined in such a way that the inner connectivity within each of them is maximized and the connectivity between sub-networks is minimized (see Fig. 2d, e). The difference between this procedure and a traditional modularity algorithm is that the sub-networks that are identified in here must have a similar number of nodes, instead of optimal sub-networks identification. The partition of the network was performed using gpmetis (Karypis and Kumar 1998) from the Metis 5.0.2 software (http://glaros.dtc.umn.edu/gkhome/metis/). Metis is a serial graph partitioning software from the Karypis $L a b$. This software allows partitioning a graph by maximizing the inner communities connectivity. 
In each network partition, for each sub-network, the $\mathrm{W}(\mathrm{K})$ relation is computed as for pRS. In contrast with the computation of $\mathrm{pRS}$, for tRS $\mathrm{W}$ represents the number of links that cross the boundary of the sub-network, and $\mathrm{K}$ represents the number of nodes within the sub-network. A power-law distribution indicates the existence of a tRS (see Fig. 2f). $B$ is called topological Rent's Exponent (tRE) which is calculated as the slope of $\mathrm{W}(\mathrm{K})$ in the log-log scale in the linear regime, using least-squares fitting.

The computation of tRE follows an optimization process that maximizes the within sub-network connectivity and minimizes the between sub-networks connectivity. Thus the $\mathrm{W}(\mathrm{K})$ relation has minimum slope, and therefore tRE is the minimum reachable value for pRE. Having this in consideration, for a given network topology, the minimum Rent's Exponent (mRE) is defined as the most efficient possible physical placement of the networks' nodes and is theoretically related to tRE, as follows (Verplaetse et al. 2001):

$m R E=\max \left(1-\frac{1}{D_{E}}, t R E\right)$

The Euclidean dimension $\left(\mathrm{D}_{\mathrm{E}}\right)$ is three, and hence mRE is equal to tRE when the latter is larger than $1-\frac{1}{D_{E}}=0.67$, or 0.67 otherwise. pRE provides a tool for quantifying the wiring efficiency of a network in the Euclidean space: the closer pRE is to the minimum expected value (mRE), the more optimized the network of connections is (Bassett et al. 2010).

Furthermore, network randomization allows computing random Rent's Exponents. These exponents will be used to estimate how far is the organization of our networks from that of a random network. The networks were one hundred times randomized in order to compute the random physical (rpRE) and topological (rtRE) Rent's Exponents. The randomization consisted on a rewiring of the links with a preservation of the nodes degree, i.e. the number of links connected to a node remains the same after the randomization.

\section{Network Directionality}

The ND $\left(N D_{i j}\right)$ is a network metric proposed here, which quantifies the magnitude of the strength of the networks' connections in the three axes X, Y, Z. Each of the axes represents one orientation in the brain network. $\mathrm{X}$ is the $\mathrm{IH}$ orientation, $\mathrm{Y}$ is anterior-posterior (AP), and $\mathrm{Z}$ is dorsalventral (DV). The ND is based on a weighted average of the direction cosines of the existent connections in the network. The ND between two nodes $\left(N_{i}, N_{j}\right)$ with Cartesian coordinates $\left[x_{i}, y_{i}, z_{i}\right]$ and $\left[x_{j}, y_{j}, z_{j}\right]$, and with a connection weight $\mathrm{w}_{\mathrm{ij}}$ is computed as follows:

$$
\begin{aligned}
N D_{i j}= & {\left[\begin{array}{l}
I H_{i j} \\
A P_{i j} \\
D V_{i j}
\end{array}\right] } \\
= & {\left[\begin{array}{l}
\left|x_{i}-x_{j}\right| w_{i j} / \sqrt{\left(x_{i}-x_{j}\right)^{2}+\left(y_{i}-y_{j}\right)^{2}+\left(z_{i}-z_{j}\right)^{2}} \\
\left|y_{i}-y_{j}\right| w_{i j} / \sqrt{\left(x_{i}-x_{j}\right)^{2}+\left(y_{i}-y_{j}\right)^{2}+\left(z_{i}-z_{j}\right)^{2}} \\
\left|z_{i}-z_{j}\right| w_{i j} / \sqrt{\left(x_{i}-x_{j}\right)^{2}+\left(y_{i}-y_{j}\right)^{2}+\left(z_{i}-z_{j}\right)^{2}}
\end{array}\right] }
\end{aligned}
$$

The result of this equation is a vector of three components $\left[I H_{i j}, A P_{i j}, D V_{i j}\right]$ that represents the contribution of each link to each of the Cartesian direction of this link. It is important to mention that this metric does not measure causality in the connection between nodes $\left(N_{i}, N_{j}\right)$, but just the orientation of the vector that links these two nodes weighted by $\mathrm{w}_{\mathrm{ij}} \cdot N D_{i j}$ was averaged for all links in the network as:

$N D=\frac{1}{N(N-1) / 2} \sum_{i, j \in N, j>i} N D_{i j}$

metric permits to integrate the position of the networks' nodes in the space with the strength of the connections in a framework that allows us to observe the brain as a unique oriented vector. ND was computed for different network densities by performing proportional thresholds.

\section{Results}

\section{Rentian Scaling}

The Rent's Exponents that were described in the "Materials and Methods" section were obtained for both AC and rsFC networks. In the distribution of tRE, two subjects of the sample were rejected. The reason for that was that the graph partitioning software did not succeed in finding optimal topological sub-network partitions for the rsFC networks of both subjects, which resulted in tRE larger than pRE. Table 1 shows the Rent's Exponents obtained for the eighteen subjects in the remaining sample (mean values and standard deviations). Unpaired $t$ test showed non-significant differences between network modalities in tRE, mRE, rpRE and rtRE. The pRE showed significant differences between $\mathrm{AC}$ and $\mathrm{rsFC}$ networks $(p<0.05$ Bonferroni-corrected), being higher for the rsFC networks. Although this result already points out to a better embedding optimization in the $\mathrm{AC}$ networks, the pRE by itself must be handled carefully, as it is necessary to compare it with the mRE and rpRE for providing confidence in the result. We computed the distances between these parameters (see Table 1) and observed that the distributions of these distances were significantly 
Table 1 Rentian scaling with $10 \%$ of the highest connections

\begin{tabular}{llll}
\hline & AC networks & rsFC networks & $p$ value \\
\hline tRE & $0.7066(0.0245)$ & $0.7180(0.0419)$ & 0.3381 \\
mRE & $0.7066(0.0245)$ & $0.7207(0.0360)$ & 0.1889 \\
pRE & $\mathbf{0 . 7 9 3 0 ( 0 . 0 2 7 6 )}$ & $\mathbf{0 . 8 6 1 4}(\mathbf{0 . 0 6 8 3})$ & $\mathbf{4 . 0 3 0 8 e - 4}$ \\
rtRE & $0.8660(0.0069)$ & $0.8648(0.0064)$ & 0.6158 \\
rpRE & $0.8997(0.0136)$ & $0.8984(0.0098)$ & 0.7411 \\
pRE-mRE & $\mathbf{0 . 0 8 6 4}(\mathbf{0 . 0 3 7 6})$ & $\mathbf{0 . 1 4 0 7}(\mathbf{0 . 0 6 6 6})$ & $\mathbf{0 . 0 0 5 2}$ \\
rpRE-pRE & $\mathbf{0 . 1 0 6 7}(\mathbf{0 . 0 3 4 8})$ & $\mathbf{0 . 0 3 7 0}(\mathbf{0 . 0 6 7 7})$ & $\mathbf{4 . 7 8 6 6 e - 4}^{\mathbf{a}}$ \\
rtRE-tRE & $0.1594(0.0252)$ & $0.1468(0.0439)$ & 0.3119 \\
\hline
\end{tabular}

Bold values are statistically significant $(p<0.01)$ after unpaired $t$ test The Rent's Exponents are the result of the Rentian Scaling analyses. The results for the AC (rsFC) networks are included in the first (second) column of the table. The third column shows the $p$ values of an unpaired $t$ test for rejecting the null hypothesis where the Rentian metrics are equally distributed for $\mathrm{AC}$ and $\mathrm{rsFC}$ networks

$t R E$ topological Rent's exponent, $m R E$ minimum Rent's exponent, $p R E$ physical Rent's exponent, rtRE random topological Rent's exponent, $r p R E$ random physical Rent's exponent

a $p$ value survives Bonferroni correction for multiple comparisons

different for the different networks $(p<0.05$ Bonferronicorrected). The distance between pRE and mRE was lower for the AC networks confirming or previous hypothesis that the organization for the $\mathrm{AC}$ networks was more optimized in the embedding space than rsFC networks.

Another factor of interest is that from the less efficient organization of the networks, i.e. random organization. We computed the distances between tRE and rtRE and between pRE and rpRE. We observed that there were non-significant differences between AC-rsFC networks for the former distances (tRE-rtRE) $(p=0.3119)$, whereas for the latter (pRE-rpRE) the AC networks showed a higher distance from a random configuration, indicating that $\mathrm{AC}$ networks were in a state of organization further from random compared to the rsFC networks $(p<0.05)$.

\section{Network Directionality}

The arrangement of the network' connections was analysed with the ND metric, which was described in the "Materials and Methods" section. In Fig. 3a, b are depicted AC and rsFC networks from a single subject over a brain template. The networks were proportionally thresholded, leaving just for representation the ten per cent of the links. It can be appreciated that both networks (AC and rsFC) differ in their spatial arrangement of connections. While most of the connections in the AC networks are contained within brain hemispheres, in the rsFC there is a higher number of $\mathrm{IH}$ connections. For quantifying this observation we computed the ND for both AC and rsFC networks.
The ND was computed for a range of proportional thresholds and the results are represented in Fig. 3c, d. It is observed that the major orientation of the connections in $\mathrm{rsFC}$ is $\mathrm{IH}$, the second major orientation is $\mathrm{AP}$ and then DV. When the $80 \%$ of the connections is eliminated the contribution of $\mathrm{IH}$ connections in the arrangement of the network starts growing exponentially, and contributions from $\mathrm{AP}$ and $\mathrm{DV}$ orientations are diminished. In the $\mathrm{AC}$ networks, contributions from the AP and DV orientations to the ND were the highest. The three components of the ND evolved at the same rate for the different proportional thresholds.

The number of slices in AP is larger than it is in IH or DV directions, thus the probability of finding a larger contribution to AP in the ND metric is the highest. In order to control this effect we computed a normalized variation of the ND metric, the normalized ND (NDn). The computation of the NDn consisted on dividing the original ND by a constant vector. This vector is the ND obtained for a fully connected network of equal weights, which indicates that all connections in the brain exist with equal probability (e.g. $w_{i j}=1 ; i, j \in N$. After correcting for this dimensional limitation, the NDn show a more realistic spatial arrangement of the connections. In Fig. 3e, f is represented the evolution of the NDn for AC and rsFC networks for a range of proportional thresholds. We observed that AP and DV contributions have been interchanged. However, the IH contributions remain unaltered, reinforcing the robustness of this result.

\section{Discussion}

Traditionally, in macroscopic brain networks characterization, concepts such as orientation of links or position of nodes are not considered as relevant, but it seems clear that these features must be of high importance in the description of the organization. In this paper different communication mechanisms considering the embedding dimensions of the brain networks were studied.

The existence of RS in a complex network is a representation of a cost-efficient spatial embedding or wiring efficiency (Bassett et al. 2010). Our results indicated that AC networks presented a higher wiring efficiency compared to rsFC networks. However, both $\mathrm{AC}$ and $\mathrm{rsFC}$ networks showed a degree of optimization, as their pRE and tRE were lower than their random counterparts rpRE and rtRE. The organization in rsFC networks was closer to random than in AC networks. In biological networks, randomness is a signature of homogeneity, since it indicates that all nodes had, in principle, equal probability of acquiring a certain property (e.g., degree, clustering, 


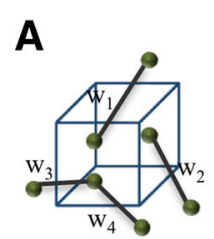

$\mathrm{K}=3 ; \mathrm{W}=\Sigma_{\mathrm{i}=1: 4} \mathrm{~W}_{\mathrm{i}}$

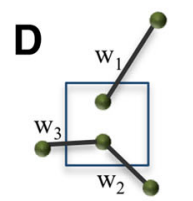

$\mathrm{K}=2 ; \mathrm{W}=\Sigma_{\mathrm{i}=1: 3} \mathrm{~W}_{\mathrm{i}}$
B

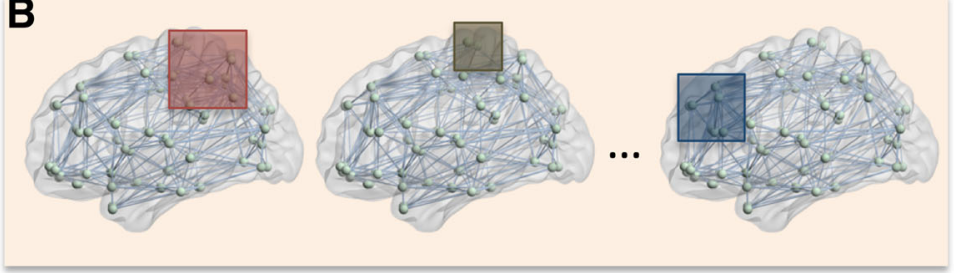

E

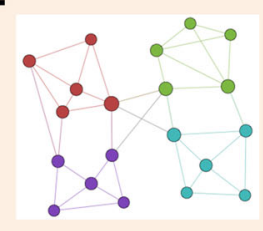

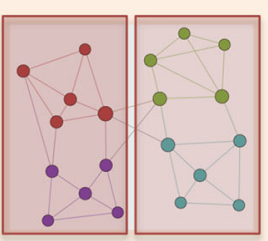

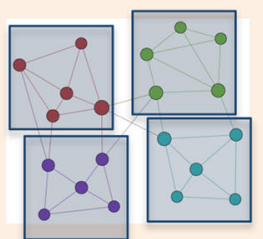

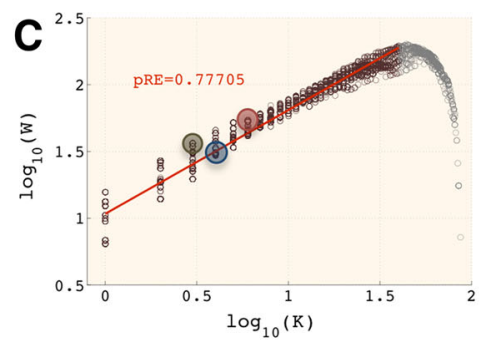

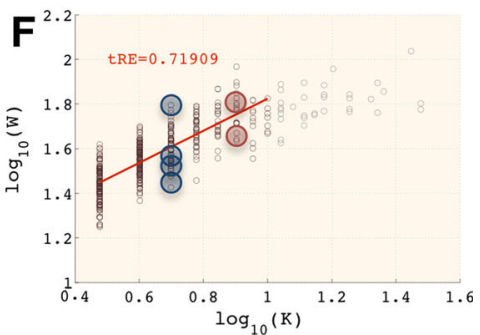

Fig. 2 Process of finding the physical $(\mathbf{a}-\mathbf{c})$ and topological (df) Rentian Scaling (RS) and Rent's exponents. a and d depict one single iteration of the physical and topological RS computation respectively. Three iterations of the scaling are performed in $(\mathbf{b}, \mathbf{e}) . \mathbf{c}$, f depict the resulting RS after $\mathrm{L}$ iterations with $\log -\log$ scaling. Grey dots represent a non-linear region, which is not considered for determining the Rent's exponent. Brain network's plots were generated using the BrainNet Viewer (Xia et al. 2013)
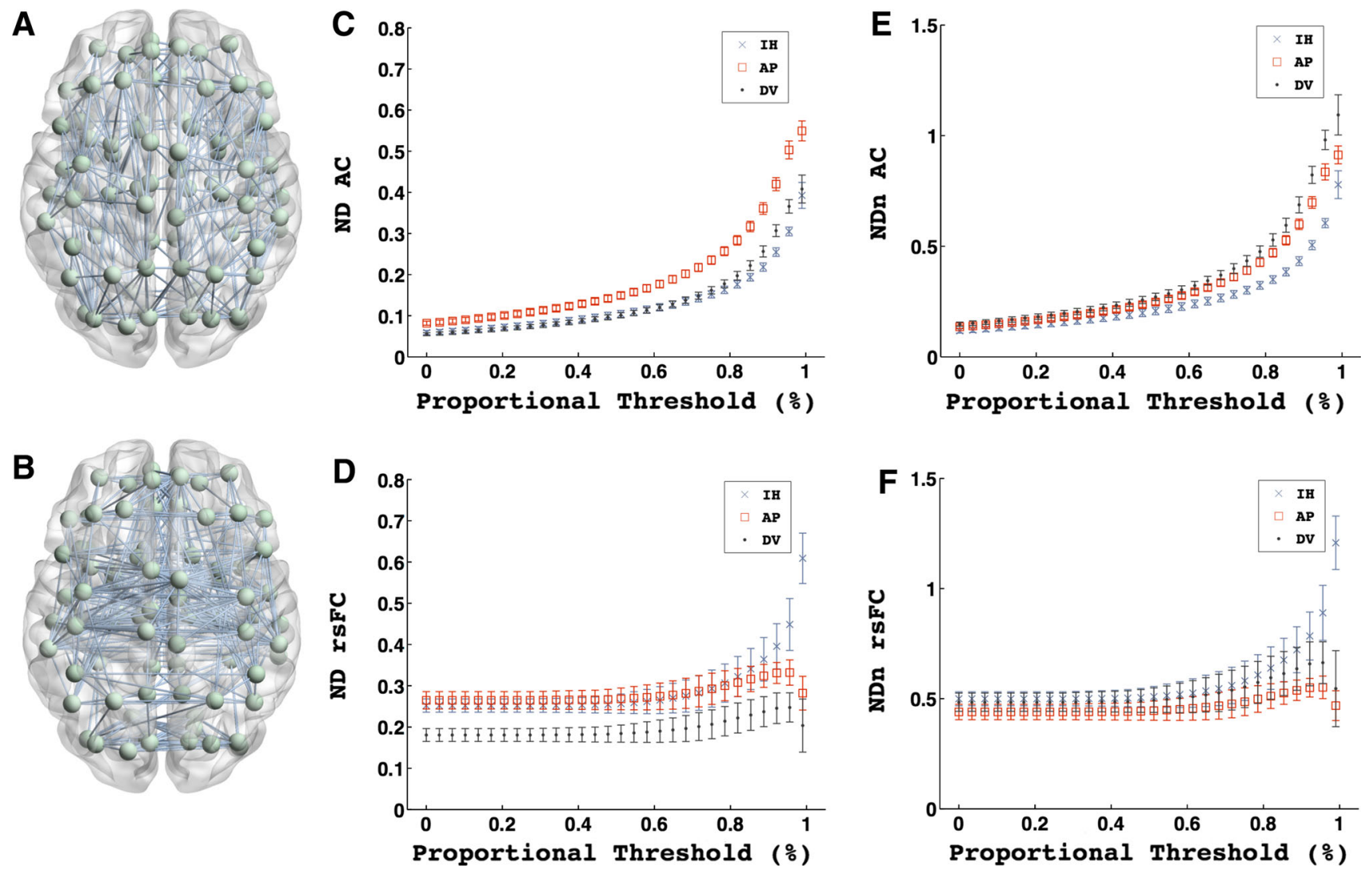

Fig. 3 Network directionality. a, b Graphs with the $8 \%$ of the strongest links of $\mathrm{AC}(\mathbf{a})$ and $\mathrm{rsFC}(\mathbf{b})$. c, d Plots of the Network Directionality (ND) for AC (c) and $\mathrm{rsFC}(\mathbf{d})$ networks versus the proportional thresholds (percentage of links deleted) that have been applied to the matrices. e, f Plots of the normalized Network
Directionality (NDn) for AC (e) and $\mathrm{rsFC}(\mathbf{f})$ networks versus the proportional thresholds that have been applied to the matrices. $I H$ inter-hemispherical, $A P$ anterior-posterior, $D V$ dorsal-ventral. Brain network's plots were generated using the BrainNet Viewer (Xia et al. 2013) 
shortest path...). Therefore, any deviation from a random structure is indicating an additional driving force that promotes/reduces the role that certain nodes play in the network, thus introducing heterogeneity. This is somehow logical, as the only limitation for the creation of a functional connection is the existence of an anatomical connection. All nodes, directly or indirectly connected by an anatomical link, will have a non-zero probability of being functionally connected. Thus, making the functional network to be driven by random forces.

These findings are not consistent with prior studies which concluded that the anatomical networks obtained by cortical volume were more random than the rsFC networks (Hosseini and Kesler 2013). One possible explanation for this difference is that the anatomical networks described by Hosseini and Kesler (2013) were obtained using cortical volume correlations, a more indirect measure than diffusion MRI tractography. The estimation of AC through correlations in such a sample size (36 subjects) may lead to an inaccurate representation of the AC. That is, this sample size could yield an increase in the false positive rate, resulting in spurious anatomical connections, and thus increasing the randomness in the network topology.

It is clear that the physical arrangement of neural nodes and their associated edges influences the effectiveness and spatial pattern of their dynamics at all scales. Thus, we consider that a quantitative characterization of the spatial arrangement of the connections could be of great interest.

The ND metric that we are proposing here, demonstrates important differences in the properties of $\mathrm{AC}$ and $\mathrm{rsFC}$ networks. While the former shows a higher contribution of AP and DV connections, the latter shows a clearly higher contribution of IH connections. These results are in concordance with previous findings (Hermundstad et al. 2013), where IH rsFC was found to present strong weights independently of the AC, which they attributed to a high myelinization in the IH anatomical streamlines. They also found that long intra-hemispheric connections presented weak values of rsFC. (Skudlarski et al. 2008) also observed these strong rsFC, and they attributed it to the vascular symmetry of the brain.

Fair et al. (2008) observed that functional IH connections between homotopic cortical regions were stronger in children than in adults, but other linkages were significantly weaker. In another study of ageing and connectivity, Andrews-Hanna et al. (2007) showed a reduced rsFC between anterior and posterior components of the default mode network in older subjects. These findings indicate that spatial arrangement of $\mathrm{rsFC}$ is related to maturation (Fair et al. 2008) and to degeneration (Andrews-Hanna et al. 2007), and hence we postulate that the use of the ND could help to characterize these developmental processes (Dosenbach et al. 2010).
Here, the proposed organizational characterization tools point to the necessity of including the location of the brain nodes in the network analysis. By doing this, we are revealing different properties between $\mathrm{AC}$ and $\mathrm{rsFC}$ networks, showing a better spatial embedding for $\mathrm{AC}$ networks and differences in the orientation of the higher weighted links. These results represent, apart from being complementary, a more complete description of the network's organization, than the characterization of the networks topologies (Hosseini and Kesler 2013).

Nevertheless, there are limitations to the current results. First, the quantification of AC using DTI-tractography is prone to errors. Fiber crossing, bending or kissing are unresolved issues when using single DTI as a model of water diffusion. More complex models, such as q-ball (Tuch 2004) or spherical deconvolution (Tournier et al. 2004) are potentially useful alternatives. However, the limited number of encoding directions in our data hinders this estimation. Future MRI protocols will include higher angular resolution diffusion schemes in order to achieve a better characterization of the structural connectivity. Second, the estimation of the topological Rent's exponents is very sensitive to artifactual connectivity values. In the sample under study, after establishing a specific connectivity threshold, we rejected two of twenty subjects because the topological RS of their functional networks wasn't sensible. If the sub-network topological partitioning algorithm does not succeed, the connectivity between subnetworks will be very high and thus the slope in the RS power law, i.e. the Rent's exponent, might also take larger values than the physical Rent's exponent. This result is theoretically impossible (Christie and Stroobandt 2000; Bassett et al. 2010). Future research should study the effect of using different pre-processing pipelines in the estimation of the topological RS. Third, the results that were obtained with the ND in macroscopic networks must be interpreted with caution. The ND metric assumes that the connection between two nodes follows a linear trajectory connecting the centroids of two regions. This is indeed not true, as the flow of information will follow the pathways defined by the WM tracts. This assumption is made in order to simplify the study of the organization of the brain networks, and further analyses are required to study the contribution of the real pathways into the ND metric. Additionally to the study of macroscopic brain networks, the ND could also be applied to the analysis of microscopic neural networks (functional or anatomical), allowing the study of brain hierarchies through definition of a net flow of connectivity in partitions of the network. Fourth the analysis of rsFC is very sensitive to physiological noise, such as respiratory, or cardiac and to head motion. Here we have modeled these noise sources by extracting the mean time series in CSF and WM masks, and together with the motion correction 
residuals we have regressed it out from the regional time series. However more accurate pre-processing techniques such as Independent Component Analysis artifacts identification or the use of physiological-noise regressors (Chang and Glover 2009) obtained from gating acquisitions will be tested in future analyses. In addition, fMRI is less representative of neural activity than other functional techniques such as electroencephalography (EEG) or magnetoencephalography (MEG), which also provide a higher temporal resolution. Therefore, remains to reproduce these results for rsFC networks, with those obtained with EEG/ MEG data.

\section{Conclusion}

Characterization of brain networks is not a unique modality approach. Both anatomical and functional networks must be studied simultaneously for a deeper understanding of brain evolving processes: maturation, plasticity and degeneration. Also, knowledge about disconnection syndromes such as has been hypothesized for Alzheimer's Disease, ADHD or Schizophrenia will benefit from a multi-modal study (Bassett and Bullmore 2009; Fornito and Bullmore 2010). We have demonstrated here that healthy AC and rsFC networks are different in the organization profiles. While the AC networks are more efficient in terms of links placement, the rsFC shows a more random configuration. The current evidence states that randomness and organization in the macroscopic brain networks are representative of cognition and pathology (Sporns 2011; Bullmore and Sporns 2012). Future studies should provide a deeper characterization with larger and more heterogeneous samples.

Acknowledgments The authors are grateful to the research participants for their participation in this study. We also thank Yasser Iturria-Medina for providing the tractography scripts. We also thank Frank G. Hillary and Nazareth P. Castellanos for fruitful comments on the manuscript. José Á. Pineda-Pardo was supported by the Spanish Ministry of Education through the National Program FPU (grant number AP2010-1317).

Disclosure No competing financial interests exist.

\section{References}

Andrews-Hanna JR, Snyder AZ, Vincent JL et al (2007) Disruption of large-scale brain systems in advanced aging. Neuron 56:924-935. doi:10.1016/j.neuron.2007.10.038

Ashburner J (2007) A fast diffeomorphic image registration algorithm. Neuroimage 38:95-113. doi:10.1016/j.neuroimage.2007.07.007

Bassett D, Bullmore E (2009) Human brain networks in health and disease. Curr Opin Neurol 22:340-347. doi:10.1097/WCO. 0b013e32832d93dd.Human

Bassett DS, Greenfield DL, Meyer-Lindenberg A et al (2010) Efficient physical embedding of topologically complex information processing networks in brains and computer circuits. PLoS Comput Biol 6:e1000748. doi:10.1371/journal.pcbi.1000748

Boorman ED, O'Shea J, Sebastian C et al (2007) Individual differences in white-matter microstructure reflect variation in functional connectivity during choice. Curr Biol 17:1426-1431. doi:10.1016/j.cub.2007.07.040

Bullmore E, Sporns O (2009) Complex brain networks: graph theoretical analysis of structural and functional systems. Nat Rev Neurosci 10:186-198. doi:10.1038/nrn2575

Bullmore E, Sporns O (2012) The economy of brain network organization. Nat Rev Neurosci 13:336-349. doi:10.1038/ $\operatorname{nrn} 3214$

Camchong J, MacDonald AW, Bell C et al (2011) Altered functional and anatomical connectivity in schizophrenia. Schizophr Bull 37:640-650. doi:10.1093/schbul/sbp131

Chang C, Glover GH (2009) Effects of model-based physiological noise correction on default mode network anti-correlations and correlations. Neuroimage 47:1448-1459. doi:10.1016/j.neuro image.2009.05.012

Christie P, Stroobandt D (2000) The interpretation and application of Rent's rule. IEEE Trans Very Large Scale Integr Syst 8: 639-648. doi:10.1109/92.902258

Cohen MX, Elger CE, Weber B (2008) Amygdala tractography predicts functional connectivity and learning during feedbackguided decision-making. Neuroimage 39:1396-1407. doi:10. 1016/j.neuroimage.2007.10.004

Collin G, Sporns O, Mandl RCW, van den Heuvel MP (2013) Structural and functional aspects relating to cost and benefit of rich club organization in the human cerebral cortex. Cereb Cortex. doi:10.1093/cercor/bht064

Damoiseaux JS, Greicius MD (2009) Greater than the sum of its parts: a review of studies combining structural connectivity and resting-state functional connectivity. Brain Struct Funct 213: 525-533. doi:10.1007/s00429-009-0208-6

Dijkstra EW (1959) A note on two problems in connexion with graphs. Numer Math 1:269-271. doi:10.1007/BF01386390

Dosenbach NUF, Nardos B, Cohen AL et al (2010) Prediction of individual brain maturity using fMRI. Science 329:1358-1361. doi:10.1126/science. 1194144

Fair DA, Cohen AL, Dosenbach NUF, et al. (2008) The maturing architecture of the brain's default network. Proc Natl Acad Sci USA 105:4028-32. doi: 10.1073/pnas.0800376105

Fornito A, Bullmore ET (2010) What can spontaneous fluctuations of the blood oxygenation-level-dependent signal tell us about psychiatric disorders? Curr Opin Psychiatry 23:239-249. doi:10.1097/YCO.0b013e328337d78d

Gong G, He Y, Concha L, Lebel C, Gross DW, Evans AC, Beaulieu C (2009) Mapping anatomical connectivity patterns of human cerebral cortex using in vivo diffusion tensor imaging tractography. Cereb Cortex 19(3):524-536. doi:10.1093/cercor/bhn102

Greicius MD, Supekar K, Menon V, Dougherty RF (2009) Restingstate functional connectivity reflects structural connectivity in the default mode network. Cereb Cortex 19:72-78. doi:10.1093/ cercor/bhn059

Guye M, Parker GJ, Symms M et al (2003) Combined functional MRI and tractography to demonstrate the connectivity of the human primary motor cortex in vivo. Neuroimage 19:1349-1360. doi:10.1016/S1053-8119(03)00165-4

Guye M, Bettus G, Bartolomei F, Cozzone PJ (2010) Graph theoretical analysis of structural and functional connectivity MRI in normal and pathological brain networks. MAGMA 23:409-421. doi:10.1007/s10334-010-0205-z

Hagmann P, Jonasson L, Maeder P, Thiran JP, Wedeen VJ, Meuli R (2006) Understanding diffusion MR imaging techniques: from scalar diffusion-weighted imaging to diffusion tensor imaging and beyond. Radiographics 26(Suppl 1):S205-S223 
Hagmann P, Cammoun L, Gigandet X et al (2008) Mapping the structural core of human cerebral cortex. PLoS Biol 6:e159. doi:10.1371/journal.pbio.0060159

Hermundstad AM, Bassett DS, Brown KS et al (2013) Structural foundations of resting-state and task-based functional connectivity in the human brain. Proc Natl Acad Sci USA 110:6169-6174. doi:10.1073/pnas.1219562110

Honey CJ, Kötter R, Breakspear M, Sporns O (2007) Network structure of cerebral cortex shapes functional connectivity on multiple time scales. Proc Natl Acad Sci USA 104:10240-10245. doi:10.1073/ pnas.0701519104

Honey CJ, Sporns O, Cammoun L et al (2009) Predicting human resting-state functional connectivity. Proc Natl Acad Sci USA 106:2035-2040

Hosseini SMH, Kesler SR (2013) Comparing connectivity pattern and small-world organization between structural correlation and resting-state networks in healthy adults. Neuroimage 78C:402-414. doi:10.1016/j.neuroimage.2013.04.032

Iturria-Medina Y, Canales-Rodríguez EJ, Melie-García L et al (2007) Characterizing brain anatomical connections using diffusion weighted MRI and graph theory. Neuroimage 36:645-660. doi:10.1016/j.neuroimage.2007.02.012

Jbabdi S, Sotiropoulos SN, Behrens TE (2013) The topographic connectome. Curr Opin Neurobiol 23:207-215. doi:10.1016/j. conb.2012.12.004

Karypis G, Kumar V (1998) Multilevelk-way Partitioning Scheme for Irregular Graphs. J Parallel Distrib Comput 48:96-129. doi:10. 1006/jpdc.1997.1404

Koch MA, Norris DG, Hund-Georgiadis M (2002) An investigation of functional and anatomical connectivity using magnetic resonance imaging. Neuroimage 16:241-50. doi: 10.1006/nimg. 2001.1052

Rubinov M, Sporns O (2010) Complex network measures of brain connectivity: uses and interpretations. Neuroimage 52:1059-1069. doi:10.1016/j.neuroimage.2009.10.003

Rykhlevskaia E, Gratton G, Fabiani M (2008) Combining structural and functional neuroimaging data for studying brain connectivity: a review. Psychophysiology 45:173-187. doi:10.1111/j. 1469-8986.2007.00621.x

Singer W (1993) Synchronization of cortical activity and its putative role in information processing and learning. Annu Rev Physiol 55:349-374. doi:10.1146/annurev.ph.55.030193.002025

Skudlarski P, Jagannathan K, Calhoun VD et al (2008) Measuring brain connectivity: diffusion tensor imaging validates resting state temporal correlations. Neuroimage 43:554-561. doi:10. 1016/j.neuroimage.2008.07.063
Skudlarski P, Jagannathan K, Anderson K et al (2010) Brain connectivity is not only lower but different in schizophrenia: a combined anatomical and functional approach. Biol Psychiatry 68:61-69. doi:10.1016/j.biopsych.2010.03.035

Smith SM (2002) Fast robust automated brain extraction. Hum Brain Mapp 17:143-155. doi:10.1002/hbm.10062

Sporns O (2011) The non-random brain: efficiency, economy, and complex dynamics. Front Comput Neurosci 5:5. doi:10.3389/ fncom.2011.00005

Takahashi E, Ohki K, Kim D-S (2008) Dissociated pathways for successful memory retrieval from the human parietal cortex: anatomical and functional connectivity analyses. Cereb Cortex 18:1771-1778. doi:10.1093/cercor/bhm204

Teipel SJ, Bokde ALW, Meindl T et al (2010) White matter microstructure underlying default mode network connectivity in the human brain. Neuroimage 49:2021-2032. doi:10.1016/j. neuroimage.2009.10.067

Tournier J-D, Calamante F, Gadian DG, Connelly A (2004) Direct estimation of the fiber orientation density function from diffusion-weighted MRI data using spherical deconvolution. Neuroimage 23:1176-1185. doi:10.1016/j.neuroimage.2004.07.037

Tuch DS (2004) Q-ball imaging. Magn Reson Med 52:1358-1372. doi:10.1002/mrm.20279

Tzourio-Mazoyer N, Landeau B, Papathanassiou D et al (2002) Automated anatomical labeling of activations in SPM using a macroscopic anatomical parcellation of the MNI MRI singlesubject brain. Neuroimage 15:273-289. doi:10.1006/nimg.2001. 0978

Van den Heuvel M, Mandl R, Luigjes J, Hulshoff Pol H (2008) Microstructural organization of the cingulum tract and the level of default mode functional connectivity. J Neurosci 28: 10844-10851. doi:10.1523/JNEUROSCI.2964-08.2008

Van den Heuvel MP, Mandl RCW, Kahn RS, Hulshoff Pol HE (2009) Functionally linked resting-state networks reflect the underlying structural connectivity architecture of the human brain. Hum Brain Mapp 30:3127-3141. doi:10.1002/hbm.20737

Verplaetse P, Dambre J, Stroobandt D, Van Campenhout J (2001) On Partitioning vs. Placement Rent Properties. Proc Intl Work Syst Interconnect Predict

Xia M, Wang J, He Y (2013) BrainNet viewer: a network visualization tool for human brain connectomics. PLoS One. doi:10.1371/journal.pone.0068910

Zhou Y, Shu N, Liu Y et al (2008) Altered resting-state functional connectivity and anatomical connectivity of hippocampus in schizophrenia. Schizophr Res 100:120-132. doi:10.1016/j. schres.2007.11.039 